\title{
Auditory Perceptual Disorder
}

National Cancer Institute

\section{Source}

National Cancer Institute. Auditory Perceptual Disorder. NCI Thesaurus. Code C84575.

A disorder characterized by the inability of the brain to properly interpret or process the auditory signals it receives from the anatomic structures of the ear. 